\title{
Focal Delivery of Neurotrophins into the Central Nervous System Using Fluorescent Latex Microspheres
}

BioTechniques 23:928-937 (November 1997)

\author{
David R. Riddle, Lawrence C. \\ Katz $^{1,2}$ and Donald C. Lo ${ }^{2}$ \\ Wake Forest University School \\ of Medicine, Winston-Salem; \\ ${ }^{1}$ Howard Hughes Medical Insti- \\ tute, ${ }^{2}$ Department of Neurobiol- \\ ogy, Duke University Medical \\ Center, Durham, NC, USA
}

\section{INTRODUCTION}

Traditionally, the biological activity of proteins and other macromolecules has been investigated by testing the effects of exogenous application of the molecules in vivo or in defined in vitro systems. Among the challenges of such experiments, particularly in vivo, are controlling the region of application and determining which cells are exposed to the factor. This issue is of particular concern in the nervous system, where neighboring populations of cells often have very different biochemical and functional properties. With most current methods for in vivo delivery of biologically active agents in the nervous system - which rely upon diffusion of an agent from the cerebral ventricle, from a slow-release polymer or from a cannula connected to an osmotic mini-pump (for example, see References 2, 3 and 19)-it is difficult to limit application to restricted populations of neurons or their terminals and to reliably identify neurons that have been exposed to the agent. In a few studies, these difficulties have been largely overcome by immobilizing the agent of interest on or within latex microspheres. Microspheres in the range of 50-200 $\mathrm{nm}$ in diameter are large enough that they diffuse only short distances within tissues, yet they are small enough that, given appropriate surface chemistry, they can be taken up by neuronal processes by endocytosis and sometimes retrogradely transported $(9,10)$. Incorporation of fluorescent dyes within such microspheres facilitates recognition of the site of applica- tion and identification of the exposed cells. Latex microspheres have been used previously to deliver carbachol to discrete regions of the pontine brain stem in studies of sleep regulation (20) and to deliver light-activated molecules to selected populations of cells to permit their destruction by photothermolysis $(12,14)$. We describe here a similar delivery system for the neurotrophins nerve growth factor (NGF), brain-derived neurotrophic factor (BDNF), neurotrophin 3 (NT-3) and neurotrophin 4/5 (NT-4/5). Microspheres coated with neurotrophins are stable and release active factor for at least 3-4 days. In vivo, the fluorescence of the microspheres provides an immediate and reliable marker of the spatial extent of neurotrophin application. Labeled microspheres remain competent to undergo endocytosis and retrograde axonal transport by neurons; thus, it is possible to apply neurotrophins to the terminal arbors of a population of neurons and examine the effects of neurotrophin treatment on their cell bodies at a distant site in the brain. This method should have utility in studying the effects of a variety of proteins in both in vitro and in vivo systems.

\section{MATERIALS AND METHODS}

\section{Preparation of Neurotrophin-Coat-} ed Microspheres

Microspheres were coated with neurotrophin by passive adsorption based on the interaction of the positively charged proteins with negatively 
charged groups of the surface of the microspheres. To remove residual surfactant and maximize the negatively charged surface available for protein binding, fluorescent latex microspheres (Lumafluor, Naples, FL, USA) were ion-exchanged for $6 \mathrm{~h}$ at room temperature with $10 \%(\mathrm{wt} / \mathrm{vol}) \mathrm{AG}^{\circledR} 501-\mathrm{X} 8$ Mixed Bed Resin (Bio-Rad, Hercules, CA, USA). The microspheres were then incubated overnight at $4^{\circ} \mathrm{C}$ with NGF (Collaborative Biomedical Products, Bedford, MA, USA), BDNF, NT3 or NT-4/5 (human recombinant BDNF, NT-3 and NT-4/5 were the gift of Regeneron Pharmaceuticals, Tarrytown, NY). For each preparation of microspheres, $20 \mu \mathrm{L}$ of microspheres (10\% solids) were added to $100 \mu \mathrm{L}$ of neurotrophin solution $(100 \mathrm{ng} / \mu \mathrm{L})$. The labeled microspheres were isolated by ultracentrifugation $\left(195000 \times g\right.$ at $4^{\circ} \mathrm{C}$ for $60 \mathrm{~min}$ ). The supernatant (containing any unbound neurotrophin) was then removed, and the microspheres were resuspended in $20 \mu \mathrm{L}$ of sterile water. The supernatant and resuspended microspheres were stored at $4^{\circ} \mathrm{C}$ until assayed in vitro or used for in vivo experiments.

\section{Analysis of Neurotrophin-Coated Microspheres In Vitro}

We used several in vitro bioassays to characterize the properties and stability of neurotrophin-coated microspheres. We initially examined the neurotrophic activity of labeled microspheres using PC12 cells stably transfected with the trkB gene (11). PC12trkB cells (gift of Regeneron Pharmaceuticals) were maintained in Dulbecco's modified Eagle medium (DMEM) supplemented with $6 \%$ horse serum, $6 \%$ fetal calf serum, $1 \%$ penicillin/streptomycin and 1\% G418 (Mediatech, Herndon, VA, USA). All cell culture reagents were obtained from Life Technologies (Gaithersburg, MD, USA) except as noted. Under these culture conditions, NGF or NT-4/5 elicited the extension of neuritic processes characteristic of neurotrophin-treated PC12 cells. Neurite extension provides a visual display of the neurotrophic activity of coated microspheres but is not easily quantified. For detailed characterization of the activity of neurotrophin-coated microspheres, we tested their ability to promote the survival of PC12trkB cells in serum-free media $(7,11)$. Cells were plated at high density (approximately $50 \%$ confluence) in 96-well plates in serum-free media. Control microspheres, neurotrophin-coated microspheres or free neurotrophin were added and the number of surviving cells was determined after $72 \mathrm{~h}$ using the CellTiter $96^{\mathrm{TM}} \mathrm{AQ}_{\text {ueous }}$ Non-Radioactive Cell-Proliferation Assay (Promega, Madison, WI, USA). We used this assay to test for direct effects of microspheres on cell survival, to estimate the concentration of neurotrophin that was carried by the microspheres and to determine the time period that the microsphere-bound neurotrophin remained active. To estimate the time period that labeled microspheres could deliver active neurotrophin at physiological temperatures, aliquots of labeled microspheres were incubated in media at $37^{\circ} \mathrm{C}$ for 1-4 days. The microspheres were removed by ultracentrifugation and resuspended; both the microspheres and the media in which they had been incubated were then tested using the survival assay.

PC12trkB cells express only TrkA and TrkB receptors and could not be used to test NT-3-coated microspheres. To verify the activity of NT-3-coated microspheres, we tested their ability to induce phosphorylation of the immediate early gene cyclic AMP response element binding protein (CREB) in a human neuroblastoma cell line. SK-N-SH cells, which express TrkB and TrkC after treatment with retinoic acid (RA; D. Lo, unpublished observations), were maintained in RPMI 1640 supplemented with $10 \%$ fetal calf serum, $1 \%$ penicillin/streptomycin and 1\% L-glutamine. The cells were plated at high density in 96-well plates and treated with $10 \mu \mathrm{M}$ RA for four days before being used for the assay. RA-treated cells were then exposed to free NT-3 or NT-3-coated microspheres for 7-60 min. As a positive control, cells were stimulated for 30-60 min with $10 \mu \mathrm{M}$ forskolin.

Phosphorylation of CREB was assayed by immunocytochemistry using a polyclonal antibody specific for the phosphorylated form of CREB (anti-
pCREB, Reference 6) obtained from Upstate Biochemical (Lake Placid, NY, USA). After stimulation as described above, the cells were fixed for $30 \mathrm{~min}$ in $4 \%$ paraformaldehyde in phosphatebuffered saline (PBS). Nonspecific binding was blocked with $5 \%$ bovine serum albumin with $0.4 \%$ Triton ${ }^{\circledR} \mathrm{X}$ 100 (BSA/TX); the cells were then incubated in the anti-pCREB antibody (diluted 1:1370 in BSA/TX) for $1 \mathrm{~h}$ at room temperature. After rinsing, the cells were incubated for $1 \mathrm{~h}$ with an alkaline phosphatase-conjugated goatanti-rabbit secondary antibody (1:500 in BSA/TX; Boehringer Mannheim, Indianapolis, IN, USA) and rinsed. The cells were then incubated with an alkaline phosphatase-labeled rabbit antibody against alkaline phosphatase (alkaline phosphatase anti-alkaline phosphatase [APAAP], 1:200; Boehringer Mannheim) for $1 \mathrm{~h}$, washed in Tris-buffered saline and finally incubated in the dark with $p$-nitrophenylphosphate (pNPP, $1 \mathrm{mg} / \mathrm{mL}$ in Tris buffer; Pierce Chemical, Rockford, IL, USA) for 1-4 h. Absorbance was read at 410 nm using a Model MR5000 Plate Reader (Dynatech, Chantilly, VA, USA).

Neurotrophins were bound to the microspheres by passive adsorption (not by covalent linking); thus, we expected they would be gradually released. We used a coculture system and the survival assay to determine whether the activity of the coated microspheres was due to the release of neurotrophin into the media or direct activation of Trk receptors on the cell membrane by microsphere-bound neurotrophin. PC12trkB cells were plated in serumfree media in 24-well plates. Polycarbonate $0.2-\mu \mathrm{m}$ pore size filter inserts (Falcon ${ }^{\circledR}$; Becton Dickinson Labware, Bedford, MA, USA) were placed in the wells, and additional media containing either free NT-4/5 (100 ng/mL) or an equivalent concentration of NT-4/5 on microspheres were added to the inserts. After three days, the filter inserts were removed, and cell survival was determined as described above. The microspheres could theoretically pass more or less freely through the filters (given their average diameter of $50 \mathrm{~nm}$ compared to the pore size of $200 \mathrm{~nm}$ ), but examination (by fluorescence microscopy) of the media above and beneath 
the filters three days after addition of microspheres revealed the vast majority of the microspheres remained above the filters, with no possibility of direct contact with the cells.

\section{Injection and Efficacy of Neurotro- phin-Coated Microspheres In Vivo}

To investigate the diffusion of neurotrophins following in vivo injection of coated microspheres, small (approximately $200 \mathrm{~nL}$ ) injections of NGF-coated microspheres were placed in the cortex of young ferrets (P40). Three to four days later, the animals were anesthetized and perfused with $4 \%$ paraformaldehyde. Frozen tangential sections of the cortex $(50 \mu \mathrm{m})$ were cut on a sliding microtome and collected in phosphate buffer. Free-floating sections were reacted to demonstrate NGF-like immunoreactivity using a rabbit-anti-NGF antibody (Collaborative Biomedical Products) visualized using the avidin-biotin-peroxidase method (Vector Laboratories, Burlingame, CA, USA). Sections were mounted onto gelatin-subbed slides, air-dried, cleared briefly in xylene and cover slips were applied.

For an independent measure of the diffusion of proteins in vivo, microspheres were coated with horseradish peroxidase (HRP; Sigma Chemical, St. Louis, MO, USA) using the same method (passive adsorption) and concentration of protein $(500 \mathrm{ng}$ per $\mu \mathrm{L}$ microspheres) used to prepare neurotrophin-coated microspheres. HRPcoated microspheres were injected into the visual cortex of two young adult rats ( 5 weeks of age). The rats were allowed to survive for one or three days, after which they were deeply anesthetized and perfused with fixative (1\% paraformaldehyde and $1.25 \%$ gluteraldehyde in phosphate buffer). Frozen sections $(50-\mu \mathrm{m}$ thick) of the visual cortex were cut on a sliding microtome and collected in buffer. Alternate sections were reacted histochemically to demonstrate peroxidase activity using diaminobenzidine (DAB; 20-min incubation in $0.05 \%$ DAB followed by 20 min additional incubation after addition of $0.01 \% \mathrm{H}_{2} \mathrm{O}_{2}$ ). The remaining sections were reacted using tetramethylbenzidine (TMB) according to the method of Mesulam (16) (20-min preincubation in $0.05 \%$ TMB in acetate buffer with $0.1 \%$ sodium nitroferricyanide, 20-min incubation after addition of $0.01 \% \mathrm{H}_{2} \mathrm{O}_{2}$ ). Following the re- action, the sections were washed in several changes of buffer, mounted onto gelatin-subbed slides, air-dried, cleared briefly in xylene and cover slips were applied.

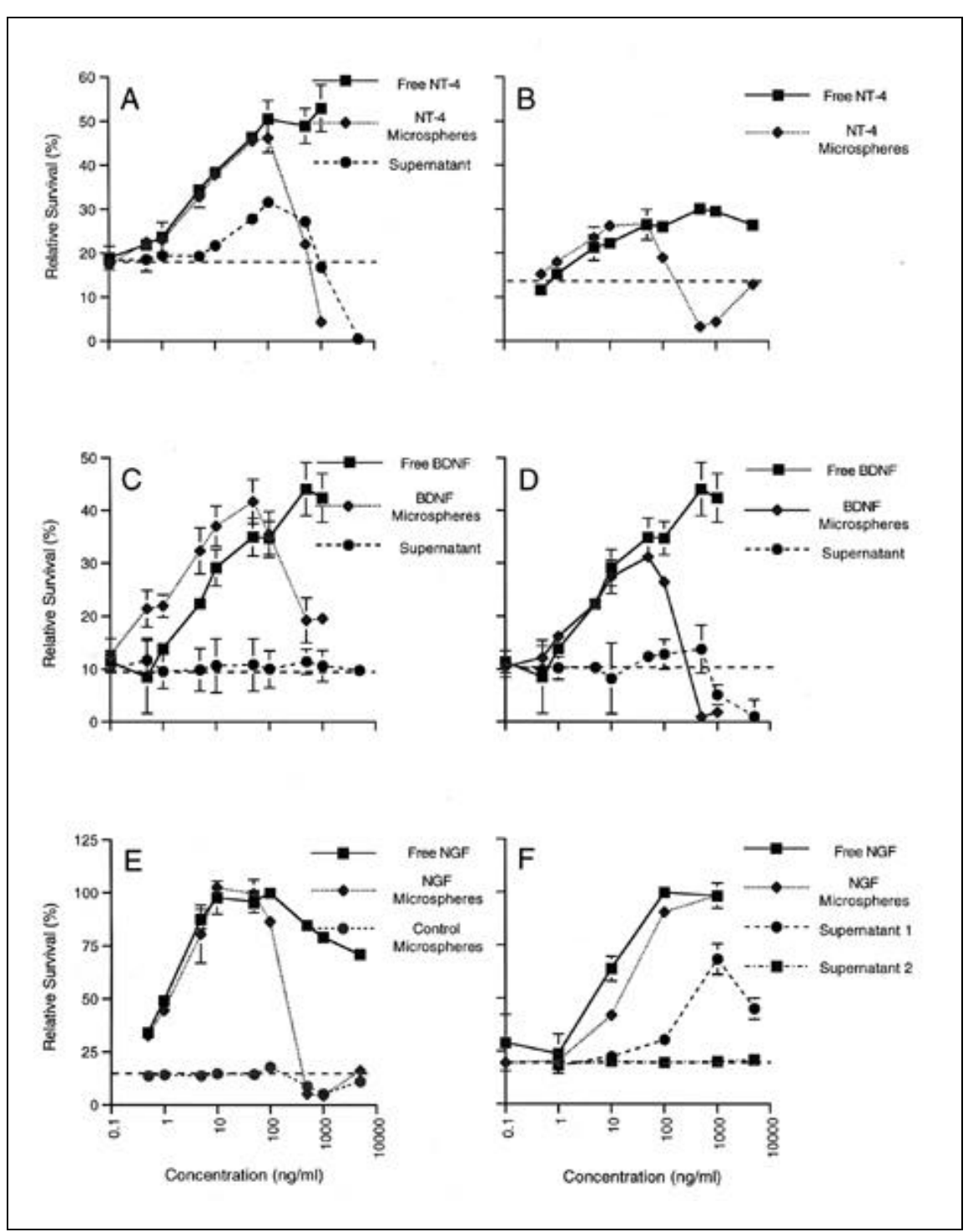

Figure 1. Efficacy of neurotrophin-coated microspheres in vitro. The plots in Panels A-F demonstrate the relative efficacy (measured as the ability to promote survival of PC12TrkB cells) of free neurotrophins, neurotrophin-coated and control microspheres, and the supernatant remaining after the preparation of coated microspheres. For free neurotrophin, data points indicate survival (relative to parallel cultures containing $100 \mathrm{ng} / \mathrm{mL}$ NGF) after addition of neurotrophin at the indicated concentration. For coated microspheres, data points indicate survival after addition of the quantity of microspheres that would have delivered the indicated concentration of neurotrophin if all of the neurotrophin in the initial preparation was bound to the microspheres. The close approximation of the dose-response curve for coated microspheres indicates virtually all of the neurotrophin was, in fact, adsorbed. The curve for control microspheres was generated by substituting equal quantities of uncoated microspheres for the neurotrophin-coated microspheres. For the supernatant, data points indicate survival after addition of the amount of supernatant that would have delivered the indicated concentration of neurotrophin if all of the neurotrophin in the initial preparation remained in the supernatant and none was adsorbed. The substantial right-shift of the growth curve (at least two log units) indicates most neurotrophin was bound to the microspheres and, in fact, only $1 \%$ or less remained in the supernatant. Two independent experiments are shown for each neurotrophin; for BDNF and NGF experiments in which the neurotrophin was carried by red (left) and green (right) microspheres are shown. Values are mean \pm standard deviation for triplicate wells. 
Microspheres were used as an in vivo delivery system to examine the effects of neurotrophins on neurons that were deprived of activity in the developing visual system. These experiments have been reported in detail elsewhere (18) and are summarized here only briefly. To determine whether neurotrophin-coated microspheres modulate the effects of monocular deprivation (MD) in the developing visual system, we closed one eye of 21 ferrets (postnatal day 38-42) and, at the same time, made highly localized injections of microspheres coated with NGF, NT-4/5, NT-3 or BDNF into the primary visual cortex (V1) of the contralateral hemisphere. The microspheres were taken up by nerve terminals and retrogradely transported; thus, after histological preparation, we could identify and measure projection neurons in the lateral geniculate nucleus (LGN) that were exposed to, bound and internalized neurotrophin. Within individual animals, adjacent injections of control microspheres permitted comparison of these neurons with others that projected to V1 but were not exposed to neurotrophin.

\section{RESULTS}

\section{Efficacy of Neurotrophin-Coated Microspheres In Vitro}

Microspheres coated with NGF, NT$4 / 5$ or BDNF promoted the survival of PC12TrkB cells in a dose-dependent manner. There was no evidence that adsorbing neurotrophins onto the surface of the microspheres diminished their activity. Neurotrophin-coated microspheres were prepared such that the maximum concentration of factor on the microspheres (given the volumes of factor and microspheres used) was 500 ng of neurotrophin per $\mu \mathrm{L}$ of microspheres. The actual concentration of each neurotrophin carried by the microspheres appeared to be close to that maximum, that is, almost all of the neurotrophin bound to the microspheres. Coated microspheres had activity approximately equal to, and sometimes exceeding, that of free neurotrophin at the concentration used to label the microspheres, while the supernatant that remained after the microspheres were coated and removed by centrifugation retained little (if any) measurable neurotrophic activity (Figure 1). In those instances in which the supernatant retained neurotrophic activity, it constituted only about $1 \%$ of the initial activity (Figure 1, A and F). Both red (rhodamine-containing) and green (fluorescein-containing) microspheres were coated with neurotrophin and tested in the survival assay. Each type of microsphere delivered neurotrophin with similar efficacy (Figure 1).

At the highest concentrations of neurotrophin delivered on microspheres, a decrease in cell survival in vitro was apparent that was not seen with equivalent concentrations of free neurotrophin. Since the microspheres were always prepared with the same concentration of neurotrophin, higher concentrations of factor were provided to the cultures by adding more microspheres. The microspheres themselves, at high concentrations, appeared to be toxic to the PC12TrkB cells. Control (uncoated) microspheres at low con- centrations had no effect upon the survival of PC12trkB cells, but when the volume of microsphere solution was $0.1 \%$ or more of the total volume of media, a decrease in cell survival was observed (Figure 1E). Therefore, cell survival at the highest concentration of neurotrophin delivered on microspheres reflects a balance between the survival-promoting effects of the factor and a detrimental effect of high concentrations of microspheres in the cultures. No evidence of toxic effects of in vivo injections of the microspheres were apparent in this study or in previous experiments that utilized the microspheres as retrograde tracers (for example, see References 9 and 10).

NT-3, either in solution or carried by microspheres, induced phosphorylation of CREB in a time-dependent manner (Figure 2). The optimal period for stimulation was approximately $15 \mathrm{~min}$ with free NT-3, but CREB phosphorylation continued to increase for $1 \mathrm{~h}$ in response to NT-3-coated microspheres.

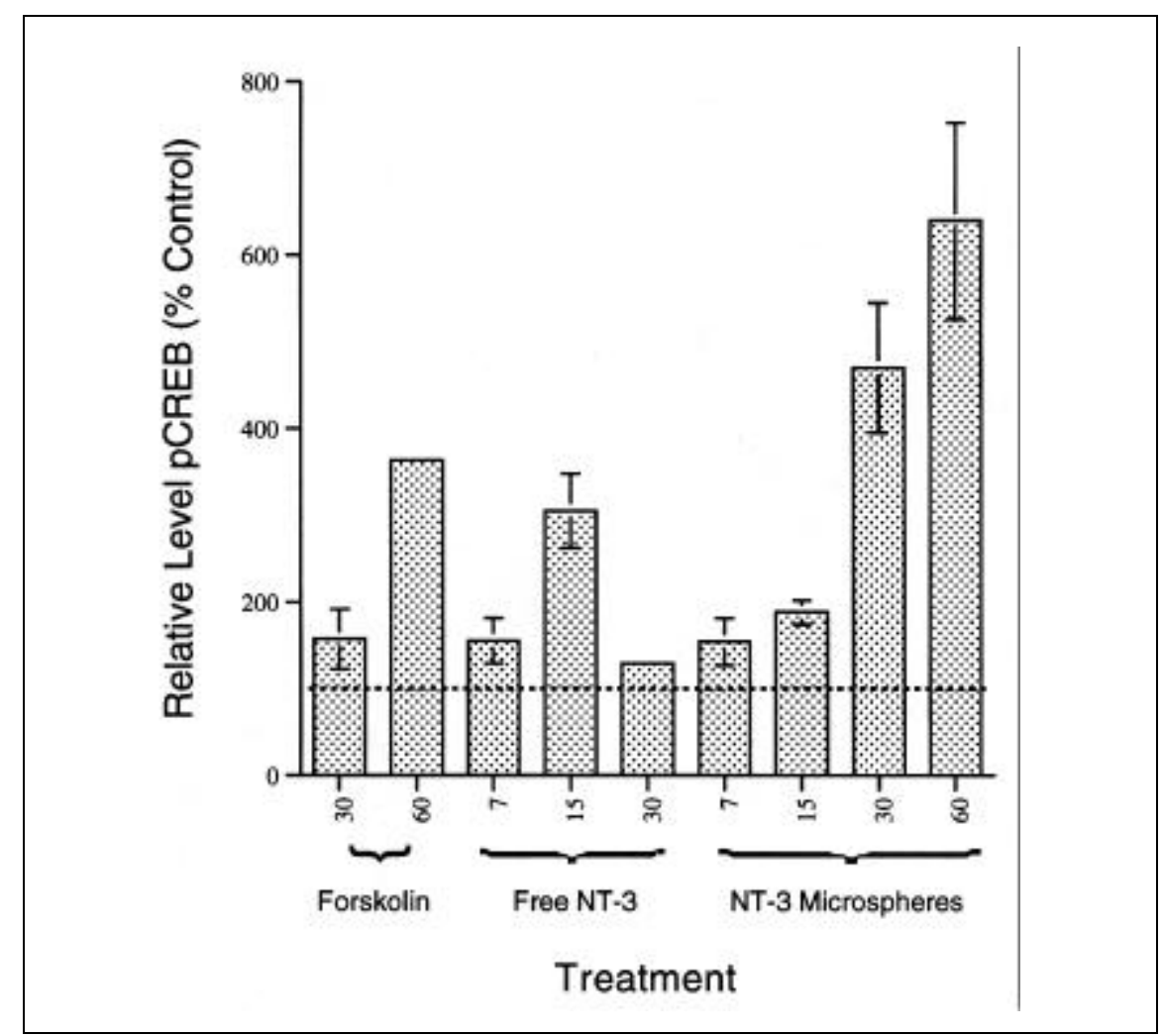

Figure 2. CREB phosphorylation in response to NT-3 and NT-3-coated microspheres. The level of phosphorylated CREB (mean \pm standard deviation relative to unstimulated parallel cultures) is shown for RA-treated SK-N-SH cells stimulated with forskolin $(10 \mu \mathrm{M})$, NT-3 $(100 \mathrm{ng} / \mathrm{mL})$ or NT-3-coated microspheres (nominal equivalent concentration $100 \mathrm{ng} / \mathrm{mL}$ ). The dashed line indicates the level of CREB phosphorylation in the absence of stimulation. 
The response to NT-3-coated microspheres appeared to exceed that to free NT-3 at an equivalent concentration (Figure 2).

NT-4/5- and NGF-coated microspheres were stored at $4{ }^{\circ} \mathrm{C}$ for at least four weeks without measurable loss of activity (Figure 3, A and B). Experiments to test the release from, and stability of, neurotrophin-coated microspheres at physiological temperatures indicated NT-4/5- and NGF-coated microspheres retained measurable neurotrophic activity after at least four days at $37^{\circ} \mathrm{C}$ (Figure $3, \mathrm{C}$ and D; BDNF and NT-3 were not tested). After four days, activity appeared to be reduced approximately two orders of magnitude.

When neurotrophin-coated microspheres and PC12TrkB cells were placed on opposite sides of polycarbonate filters (cocultures), there was no significant loss of the ability of the coated microspheres to promote cell survival, despite the fact that under these conditions all but a small percentage of the microspheres remained above the filter and out of contact with the cells. A slight $(10 \%)$ decrease in survival (relative to parallel cultures without filter inserts) was evident under these culture conditions, but the same decrease $(11 \%)$ was seen in cocultures in which free NT-4 rather than NT-4/5-coated microspheres were added. This slight loss of efficacy was probably due to binding of NT- $4 / 5$ to the filters. Taken together, these observations indicate it was not necessary for cultured cells to have direct access to labeled microspheres for the neurotrophin initially bound to the microspheres to exert its effect.

\section{Efficacy of Neurotrophin-Coated Microspheres In Vivo}

The in vitro studies confirmed the fluorescent microspheres can deliver neurotrophin that remains biologically active for at least several days at physiological temperatures. The observation that released neurotrophin could ac-

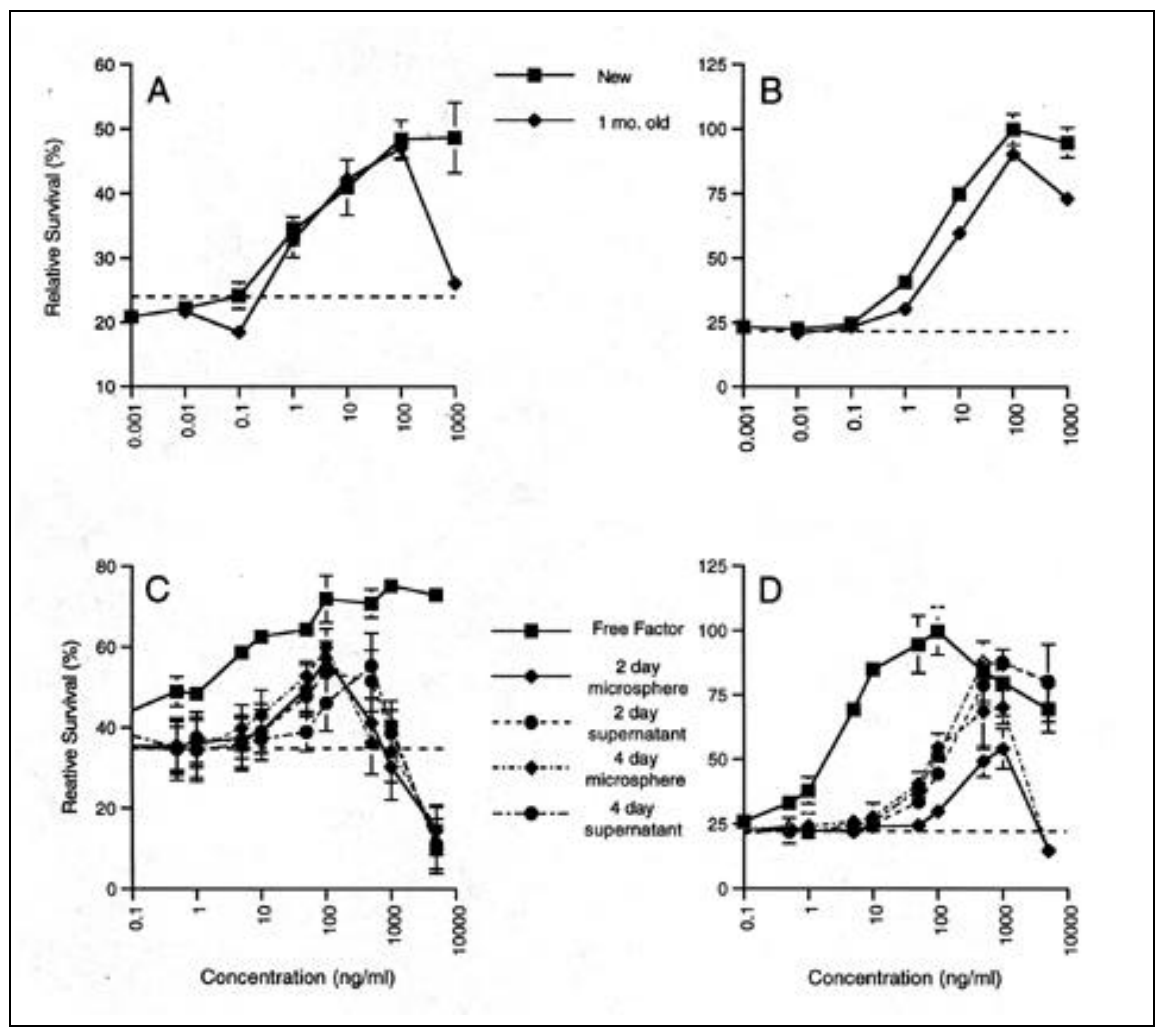

Figure 3. Stability of neurotrophin-coated microspheres. (A and B) NT-4-and NGF-coated microspheres maintained full efficacy in the survival assay after at least one month at $4^{\circ} \mathrm{C}$. $(\mathrm{C}$ and $\mathrm{D})$ Each type of coated microspheres continued to deliver active neurotrophin after at least four days at $37^{\circ} \mathrm{C}$. Decay in efficacy of neurotrophin-coated microspheres at physiological temperatures reflected both release of factor into the supernatant and some loss of activity, since activity in the supernatant was always less than the activity lost from the coated microspheres. Values are mean \pm standard deviation for triplicate wells. count for the activity in vitro led us to investigate the degree to which proteins diffuse from the microsphere injection site in vivo. NGF-like immunoreactivity (NGF-IR) was evident at injection sites containing NGF-coated microspheres but not at injections of control microspheres (Figure 4). NGF-IR was limited to the injection site; there was no evidence of significant NGF diffusion within the limits of detection. Similarly, after injection of HRP-coated microspheres, HRP activity was always coincident with the microsphere injection sites (Figure 5). There was no evidence that HRP diffused beyond the injection site, regardless of whether peroxidase activity was visualized using $\mathrm{DAB}$ or the more sensitive TMB method. Taken together, these experiments indicate there is little diffusion of protein away from the microsphere injection site. Thus, it appears that the fluorescence of the microspheres is a reliable marker of the region of neurotrophin application in vivo.

Coating the microspheres with

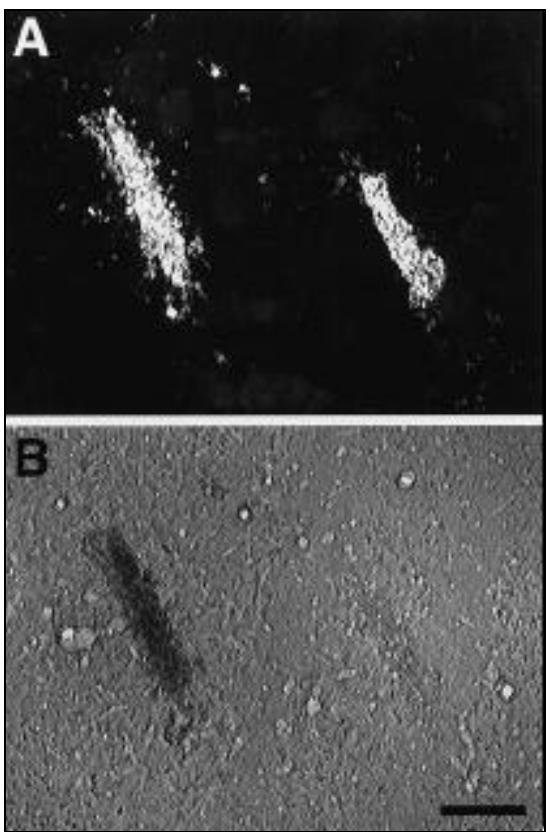

Figure 4. Diffusion of NGF from microspheres in vivo. (A) A cross-section through the ferret visual cortex is shown with adjacent injections of NGF-coated (left) and control microspheres (right). (B) Immunocytochemical labeling with an anti-NGF antibody reveals NGF-like immunoreactivity associated with NGF-coated microspheres but not control microspheres. There is no indication of significant diffusion of NGF from the injection site. Scale bar $=100 \mu \mathrm{m}$. 
protein did not interfere with the ability of neurons to take up the microspheres at their terminals and transport them retrogradely to the cell body (Figure 6). Thus, it was possible to identify neurons with terminals that were exposed to neurotrophin after in vivo injection of coated microspheres. Experiments combining microsphere injection with monocular deprivation demonstrated that neurotrophin-coated microspheres were active in vivo (18). After four days of monocular deprivation, the cell bodies of neurons in the deprived layer of the LGN labeled with control microspheres were $20 \%-30 \%$ smaller than control neurons in the adjacent region of the non-deprived layer. Cells in the deprived layer containing NT-4-coated microspheres, however, did not show such shrinkage. NT-4-coated microspheres prevented the shift in cell somal size distribution towards smaller somata that was caused by MD. The other neurotrophin-coated microspheres (NGF, BDNF or NT-3) did not block the atrophy that followed MD. Since in vitro assays demonstrated the microspheres carried these factors as effectively as NT-4, the results in vivo suggest biological specificity.

\section{DISCUSSION}

These experiments demonstrate fluorescent latex microspheres can deliver biologically active neurotrophin, mark the region to which factor was applied and label neurons whose distant terminals were exposed to neurotrophin. Not only is neurotrophic activity maintained, it appears neurotrophins delivered on microspheres may have somewhat greater efficacy than the same concentration of neurotrophin delivered free in solution, since, in some cases, coated microspheres produced a greater response than free factor at an equivalent concentration (e.g., Figure 1C) or the sum of the survival-promoting activity of coated microspheres and the supernatant remaining from their preparation exceeded the total activity expected from the neurotrophin initially used to coat the microspheres (e.g., Figure 1F). In addition to such evidence of increased efficacy in the survival assays, the level of phosphorylation of CREB obtained in response to NT-3-coated microspheres was clearly greater than that seen in response to free NT-3. Increased efficacy could arise from a stabilizing effect such that

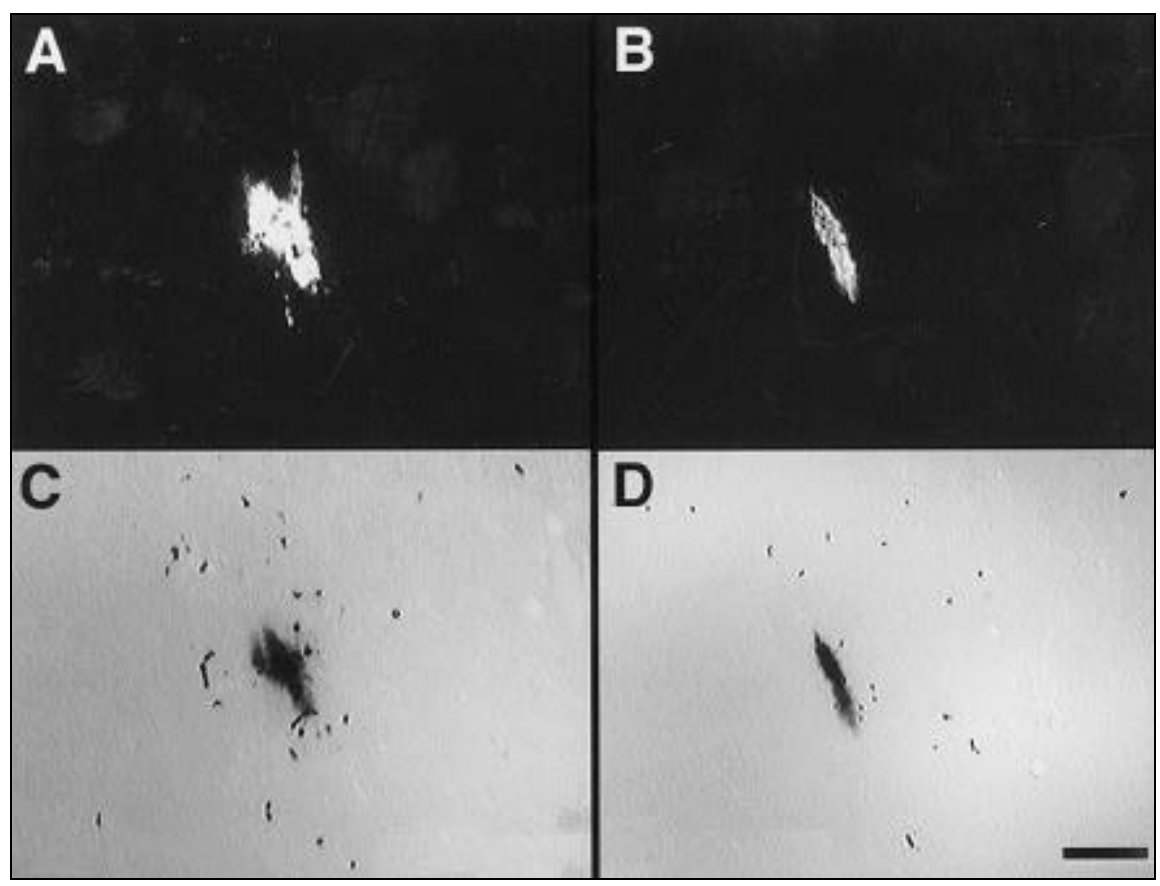

Figure 5. Diffusion of HRP from microspheres in vivo. (A and $B$ ) Cross-sections through two rat visual cortices are shown containing injections of HRP-coated microspheres. (C and D) HRP activity, demonstrated here using DAB, was limited to the injection site; there was no evidence that HRP diffused significantly from the microspheres. Scale bar $=100 \mu \mathrm{m}$. immobilization of the neurotrophin on the microspheres makes it less subject to degradation. Alternatively, the effect of a given amount of neurotrophin may be greater when the factor is supplied more gradually. The results of NT-3 stimulation of SK-N-SH cells suggests neurotrophins delivered on the microspheres are made available with a slower time course (Figure 3).

The delivery system described here provides at least two significant advantages over other methods for in vivo delivery of neurotrophins and other agents. First, although the concentration of neurotrophin delivered to neurons at any given distance from the in vivo injection site remains to be determined, it appears the microspherebased delivery system provides dramatically more focal application of neurotrophin than previously used methods. In other studies, immunocyto-

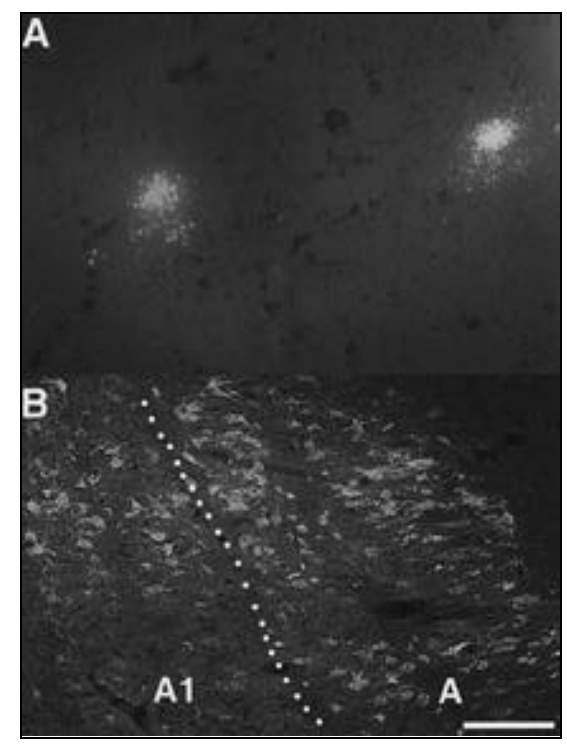

Figure 6. In vivo delivery of neurotrophincoated microspheres. (A) A tangential section through Area 17 of ferret cortex is shown containing one injection of red, NT-4-coated microspheres (which appear yellow in this double exposure) and one of green, control microspheres. (B) Groups of retrogradely labeled neurons extend across the LGN after such injections in V1 (the border between the $\mathrm{A}$ and $\mathrm{A} 1$ laminae of the LGN is indicated by the dotted line). The adjacent regions of red- and green-labeled cells projected to adjacent injection sites in the visual cortex and generally contained similar numbers of retrogradely labeled neurons. Red-labeled cells appear to predominate in this particular horizontal section, since it passes through the center of one group of cells and the margin of the other. Scale bar $=250 \mu \mathrm{m}(\mathrm{A}) ; 150 \mu \mathrm{m}$ (B). 


\section{Research Reports}

chemical and functional assays indicated neurotrophins diffuse throughout the hemisphere after intraventricular injection $(8,17)$. Immunocytochemical assays following infusion of NT-4 or NT3 by osmotic mini-pump revealed detectable neurotrophin over an area several millimeters in diameter (2). In contrast, similar immunocytochemical analysis in the present study indicated the spread of NGF was restricted to the site of microsphere injection. More importantly, we have seen effects of NT-4 treatment on one population of geniculate neurons in the absence of effects upon neighboring populations that project to adjacent sites in V1, sites separated by only a few hundred micrometers. Such limited diffusion of neurotrophins from labeled microspheres, despite the fact that neurotrophins were bound by passive adsorption (by ionic interactions) rather than by covalent coupling, is consistent with earlier reports that the conjugation of carbachol to latex microspheres significantly reduces its diffusion in vivo (13).

In addition to providing for focal delivery, the microsphere-based delivery system may decrease the amount of factor that must be delivered to produce effects in vivo. Notably, the effects we have described in vivo were produced by significantly less neurotrophic factor than has been delivered in earlier in vivo studies. Given the maximum concentration of factor carried by the microspheres and the size of the injections (approximately $200 \mathrm{~nL}$ ), a single injection of neurotrophin-coated microspheres delivered approximately $100 \mathrm{ng}$ of factor, which was sufficient to block the effects of MD. Previous studies have involved the delivery of tens of micrograms of neurotrophin, usually over days or weeks (for example, see References 1-4, 8 and 15). Due perhaps to differences in the amount of NGF used or in the method of delivery, the failure of NGF to affect geniculate neurons when delivered to their terminals in V1 in ferrets stands in contrast to previous reports that NGF injected in the ventricles blocks the effects of MD in rats and cats $(1,3,4,15)$. Since NGF infused into the ventricles influences NGF-responsive neurons throughout the brain (8), it may alter the effects of MD after such administration by acting outside of the LGN and V1, perhaps affecting modulatory circuits arising from the basal forebrain (5). The more focal delivery of neurotrophin using the present method provides greater confidence in determining the site of activity.

We have used the microspheres experimentally only for the delivery of neurotrophins, but the method should be useful for examining the effects of a variety of neuroactive factors in the intact nervous system. This study demonstrates that, at least for some proteins, 
simple adsorption onto the surface of the microspheres is sufficient to provide focal delivery of the protein and thus simplify interpretation of experimental results. While the method cannot provide the long-term, stable delivery of reagents obtained with slowrelease polymers or osmotic methods, the potentially great reduction in the amount of factor required to elicit biological effects can provide significant conservation of rare or expensive reagents. In addition, the availability of both red and green microspheres, coupled with the capacity for focal application, facilitates comparison within individual animals of treated and control neurons or of populations of neurons treated with different agents. In general, the ability to restrict the application of experimental factors to small regions of the brain and to specific populations of neurons to differentially expose specific domains of neurons (axons or dendrites, for example) and to identify treated neurons, should benefit studies in many areas of neuroscience.

\section{ACKNOWLEDGMENTS}

We thank M. Greenberg for helpful advice on the pCREB assay and $\mathrm{N}$. Tang and K. McAllister for critical review of the manuscript. Supported by grants from the NIH (D.R.R. and L.C.K.) and the Alfred P. Sloan Foundation (D.C.L.).

\section{REFERENCES}

1.Berardi, N., L. Domenici, V. Parisi, T. Pizzorusso, A. Cellerino and L. Maffei. 1993. Monocular deprivation effects in the rat visual cortex and lateral geniculate nucleus are prevented by nerve growth factor (NGF). I. Visual cortex. Proc. R. Soc. Lond. B. Biol. Sci. 251:17-23

2.Cabelli, R.J., A. Hohn and C.J. Shatz. 1995. Inhibition of ocular dominance column formation by infusion of NT-4/5 or BDNF. Science 267:1662-1666.

3.Domenici, L., N. Berardi, G. Carmignoto, G. Vantini and L. Maffei. 1991. Nerve growth factor prevents amblyopic effects of monocular deprivation. Proc. Natl. Acad. Sci. USA 88:8811-8815.

4.Domenici, L., A. Cellerino and L. Maffei. 1993. Monocular deprivation effects in the rat visual cortex and lateral geniculate nucleus are prevented by nerve growth factor (NGF). II. Lateral geniculate nucleus. Proc. R. Soc. Lond. B. Biol. Sci. 251:25-31.
5.Domenici, L., G. Fontanesi, A. Cattaneo, P. Bagnoli and L. Maffei. 1994. Nerve growth factor (NGF) uptake and transport following injection in the developing rat visual cortex. Vis. Neurosci. 11:1093-1102.

6.Ginty, D.D., A. Bonni and M.E. Greenberg. 1994. Nerve growth factor activates a Ras-dependent protein kinase that stimulates c-fox transcription via phosphorylation of CREB. Cell 77:713-725.

7.Greene, L.A. 1978. Nerve growth factor prevents the death and stimulates the neuronal differentiation of clonal PC12 pheochromocytoma cells in serum-free medium. J. Cell. Biol. 78:747-755.

8.Holtzman D.M., J. Kilbridge, Y. Li, E.T. Cunningham, Jr., N.J. Lenn, D.O. Clary, L.F. Reichardt and W.C. Mobley. 1995. TrkA expression in the CNS: evidence for existence of several novel NGF-responsive CNS neurons. J. Neurosci. 15:1567-1576.

9.Katz, L.C., A. Burkhalter and W.J. Dreyer. 1984. Fluorescent latex microspheres as a retrograde neuronal marker for in vivo and in vitro studies of visual cortex. Nature 310:498500.

10.Katz, L.C. and D.M. Iarovici. 1990. Green fluorescent latex microspheres: a new retrograde tracer. Neuroscience 34:511-520.

11.Ip, N.Y., T.N. Stitt, P. Tapley, R. Klein, D.J. Glass, J. Fandl, L.A. Greene, M. Barbacid and G.D. Yancopoulos. 1993. Similarities and differences in the way neurotrophins interact with the Trk receptors in neuronal and nonneuronal cells. Neuron 10:137-149.

12.Macklis, J.D. and R.D. Madison. 1991. Neuroblastoma grafts are noninvasively removed within mouse neocortex by selective laser activation of intracellular photolytic chromophore. J. Neurosci. 11:2055-2062.

13.Macklis, J.D. and J.J. Quattrochi. 1991. Restricted diffusion and stability of carbacholfluorescent nanospheres in vivo. Neuroreport 2:247-250.

14.Madison, R., J.D. Macklis and C. Thies. 1990. Latex nanosphere delivery system (LNDS): novel nanometer-sized carriers of fluorescent dyes and active agents selectively target subpopulations via uptake and retrograde transport. Brain Res. 522:90-98.

15.Maffei, L., N. Berardi, L. Domenici, V. Parisi and T. Pizzorusso. 1992. Nerve growth factor (NGF) prevents the shift in ocular dominance distribution of visual cortical neurons in monocularly deprived rats. J. Neurosci. 12:4651-4662.

16.Mesulam, M.M. 1978. Tetramethyl benzidinene for horseradish peroxidase neurohistochemistry: a non-carcinogenic blue reaction product with superior sensitivity for visualizing neural afferents and efferents. J. Histochem. Cytochem. 26:106-117.

17.Nawa, H., M.A. Pelleymounter and J. Carnahan. 1994. Intraventricular administration of BDNF increases neuropeptide expression in newborn rat brain. J. Neurosci. 14:3751-3765.

18.Riddle, D.R., D.C. Lo and L.C. Katz. 1995. NT-4-mediated rescue of lateral geniculate neurons from effects of monocular deprivation. Nature 378:189-191.

19.Schlaggar, B.L., K. Fox and D.D. O'Leary. 1993. Postsynaptic control of plasticity in de- veloping somatosensory cortex. Nature 364:623-626.

20.Quattrochi, J.J., A.N. Mamalek, R.D. Madison, J.D. Macklis and J.A. Hobson. 1989. Mapping neuronal inputs to REM sleep induction sites with carbachol-fluorescent microspheres. Science 245:984-986.

Received 15 May 1997; accepted 18 September 1997.

\section{Address correspondence to:}

David R. Riddle

Department of Neurobiology and Anatomy

Wake Forest University School of Medicine

Medical Center Boulevard

Winston-Salem, NC 27157-1010, USA

Internet:driddle@bgsm.edu 